SLAC-PUB-15357

January 2013

\title{
UV LASER RADIATION: SKIN HAZARDS AND SKIN PROTECTION CONTROLS ${ }^{1}$ \\ Paper \#303
}

\author{
Jeff Corbett, Michael Woods \\ SLAC National Accelerator Laboratory, \\ 2575 Sand Hill Rd., Menlo Park, CA 94025
}

\begin{abstract}
This paper examines the potential for hazardous skin exposures to ultraviolet (UV) laser radiation and provides guidance for safety controls to protect the skin. Skin hazard descriptions and control requirements related to $\mathrm{UV}$ radiation found in the ANSI laser safety standard, the OSHA Technical Manual on Laser Safety, and the ACGIH Handbook of Threshold Limit Values (TLVs) are presented. Example calculations for MPE (Maximum Permissible Exposure) and NHZ (Nominal Hazard Zone) values are shown, and some hazardous exposure incidents are described.
\end{abstract}

\section{Introduction and Overview}

Laser safety primarily focuses on eye protection, but skin protection is also important. This is especially true for UV lasers, where moderate to long exposures to diffuse reflections need to be considered.

Sections in the ANSI [1], OSHA [2] and ACGIH [3] safety documents that describe skin hazards and controls for UV exposures are noted in Appendices 13. Based on these, the following comments can be made:

- MPEs in the UV depend on the cumulative exposure. For example the MPE is $3 \mathrm{~mJ} / \mathrm{cm}^{2}$ between $180-300 \mathrm{~nm}$ for exposures from $10^{-9} \mathrm{~s}$ to 1000s. Hence, the potential for hazardous extended exposures from diffuse reflections must be considered.

- $\quad$ Eye and skin MPEs are the same in the UV (and in the far IR beyond the retinal hazard region) for exposures up to 1000s. In the retinal hazard region between 400-1400nm, eye MPEs are $\sim$ x100-1000 less than skin MPEs for a 1000s exposure.

- $\quad$ MPEs in the UV are the same for coherent (laser) and incoherent sources.

- MPEs drop by a factor of 300 at wavelengths below 300nm. This is because photochemical effects become important, meaning that chemical bonds can break resulting in risk for cancer. UV-B radiation between $280-315 \mathrm{~nm}$ seems to have the most hazardous consequences.

- Skin injuries are less serious than eye injuries. This is because vision impairment has much higher consequences and because skin injuries tend to be self-repairing.

- Skin injuries are much more probable than eye injuries. This is due to the much larger surface area, that skin PPE (personal protective equipment) may not be used as often as eyewear protection, and that hands may be close to laser beams.

- Chronic skin damage from UV laser operations is not well documented in the literature, but MPEs and injury thresholds are fairly well understood from studies with incoherent light sources, sunlight etc.

This paper is organized as follows:

- Section 2 gives examples of MPE and NHZ calculations for prolonged UV exposures.

- Section 3 provides information on recent skin injuries from lasers and from incoherent UV light sources.

- $\quad$ Section 4 gives guidance on controls for UV lasers to protect against hazardous skin exposures.

- Appendices 1-3 present information from ANSI, OSHA and ACGIH documents related to UV skin exposure.

\footnotetext{
${ }^{1}$ Contributed to the 2013 International Laser Safety Conference, Orlando, FL; http://www.lia.org/conferences/ilsc.
} 
Skin protection can best be achieved with engineering controls - enclose UV laser beam paths as much as practical! If there are open UV laser beam paths, be aware that PPE for eye and skin protection are providing primary protection to accessible diffuse reflections.

\section{MPE Values and Examples of Calculated NHZs}

MPE values for eye and skin are shown in Figure 1 for an exposure time of 1000s, which could represent a prolonged exposure to diffuse laser reflections or an incoherent source. These MPEs are the same for eye and skin outside of the retinal hazard region from 400-

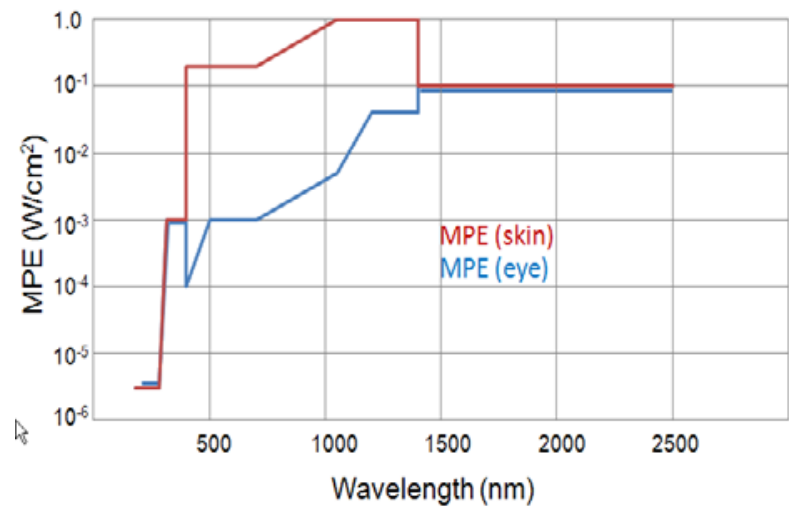

Figure 1: MPEs for eye and skin for 1000s exposure, using Tables 5a and 7 in ANSI Z136.1-2007. The MPEs are the same outside of the retinal hazard region, 400-1400nm (red and blue lines have small artificial offsets in the UV and mid-IR in the figure). 1400nm. The MPE is shown to drop by a factor of 300 in the UV region below about 300nm. Figure 2 shows more detail of the MPE in the UV, highlighting the dependence on cumulative dose.

The NHZ is a region in which exposures can exceed the MPE. Table 1 gives examples of NHZ distances for diffuse reflections from a small UV laser beam incident on a diffuse target, for an exposure duration of 1000 s. The examples assume a reflectivity of $50 \%$ for the diffuse target and a viewing angle of 45 degrees. The calculations show that for the NHZ to be $20 \mathrm{~cm}$ or greater at $380 \mathrm{~nm}$, the incident beam power on target must be at least $3.5 \mathrm{~W}$. But at $255 \mathrm{~nm}$, the same $20 \mathrm{~cm}$ NHZ occurs for only $10 \mathrm{~mW}$ incident power.

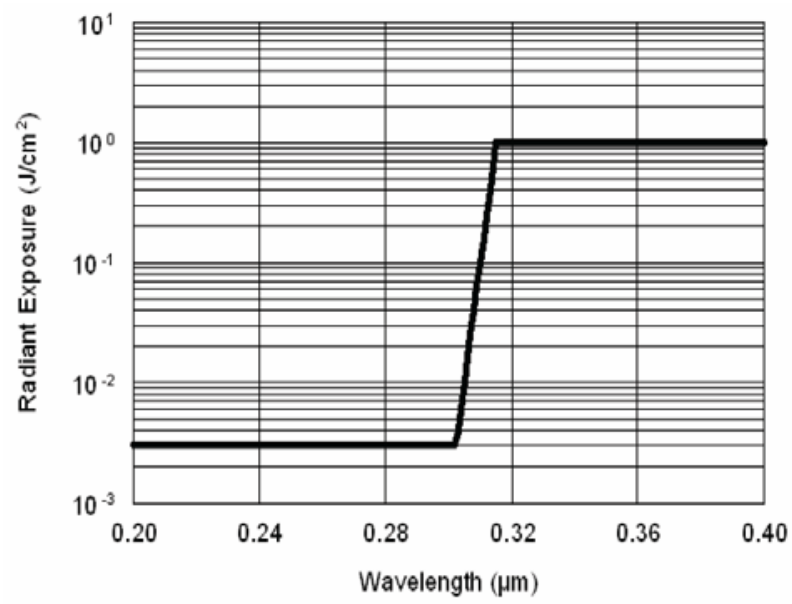

Figure 2: MPEs in the UV region for exposure durations from $10^{-9}$ to $3.10^{4}$ seconds for ocular exposure and $10^{-9}$ to $10^{3}$ seconds for skin exposure.

(Figure 5 in reference [1])

Table 1: Examples of diffuse NHZ calculations in the UV region, using EasyHaz [4]

\begin{tabular}{|c|c|c|c|c|c|c|}
\hline Wavelength & $\begin{array}{c}\text { Average } \\
\text { Power }\end{array}$ & $\begin{array}{c}\text { Diffuse } \\
\text { Target } \\
\text { Reflectivity }\end{array}$ & $\begin{array}{c}\text { Viewing } \\
\text { Angle } \\
\text { (degrees) }\end{array}$ & $\begin{array}{l}\text { Exposure } \\
\text { Duration }\end{array}$ & $\begin{array}{c}\text { MPE } \\
\left(\mathrm{W} / \mathrm{cm}^{2}\right)\end{array}$ & $\begin{array}{c}\text { Diffuse } \\
\text { Reflection } \\
\text { NHZ }\end{array}$ \\
\hline $380 \mathrm{~nm}$ & $90 \mathrm{~W}$ & $50 \%$ & 45 & 1000s & 0.001 & $1.0 \mathrm{~m}$ \\
\hline $380 \mathrm{~nm}$ & $3.5 \mathrm{~W}$ & $50 \%$ & 45 & $1000 \mathrm{~s}$ & 0.001 & $20 \mathrm{~cm}$ \\
\hline $380 \mathrm{~nm}$ & $300 \mathrm{~mW}$ & $50 \%$ & 45 & $1000 \mathrm{~s}$ & 0.001 & $6 \mathrm{~cm}$ \\
\hline $255 \mathrm{~nm}$ & $250 \mathrm{~mW}$ & $50 \%$ & 45 & $1000 \mathrm{~s}$ & $3.0 \cdot 10^{-6}$ & $1.0 \mathrm{~m}$ \\
\hline $255 \mathrm{~nm}$ & $10 \mathrm{~mW}$ & $50 \%$ & 45 & $1000 \mathrm{~s}$ & $3.0 \cdot 10^{-6}$ & $20 \mathrm{~cm}$ \\
\hline $255 \mathrm{~nm}$ & $1 \mathrm{~mW}$ & $50 \%$ & 45 & $1000 \mathrm{~s}$ & $3.0 \cdot 10^{-6}$ & $6 \mathrm{~cm}$ \\
\hline
\end{tabular}




\section{Skin Injury and UV exposure Incident Reports}

Historically, reports of skin injuries are much less than for eye injuries. Given the exposure probability, however, one expects a far greater incidence of skin injuries than eye injuries. Yet experience shows a higher rate of eye injury reports. For example, Department of Energy (DOE) laser incident reports during 2001-2012 describe 7 eye injuries and 1 hand injury [5]. In addition there are 2 DOE-reported skin injuries in this time period from non-laser exposures to UV transilluminator sources [6]. A search of Class 3B and Class 4 laser incidents (excluding DOE incident cases from 2001-2012) in the Rockwell Laser Industries (RLI) accident database [7] produces a total of 16 eye incident reports but only 4 skin incident reports since 1990. In this section we investigate sources, symptoms and reporting of skin injuries that are either laser-induced or from a UV source.

Common sources of hazardous UV radiation extend beyond conventional laser beam facilities to include large, diffuse and incoherent illumination apparatus. Examples include broadband high-brightness plasma sources for spectroscopy research, bacteria genocide systems, collateral and plasma radiation from cutting and welding processes and UV transilluminators. For sources in these categories a consistent theme among reported UV skin and eye injuries is that personnel were not fully aware of the inherent UV source hazards.

Many laboratory UV sources generate continuum radiation clear across the UV-A, B and/or C portion of the electromagnetic spectrum. Overexposure can lead to skin and eye irritation or tissue damage, with the possibility of significant delay time for symptoms relative to the incident dose. The most common reaction is thermal 'sunburn' (erythema) including rash, itch, peeling or blisters that can exhibit delayed symptoms of several hours or more. Medications such as the antibiotic tetracycline can further enhance skin sensitivity to UV light exposure. Long-term effects, in particular for chronic exposures, are also possible and laboratory workers need to be concerned with skin cancer risks as UV radiation can photochemically break bonds within living tissue.

An increasingly common source of UV overexposure is the UV transilluminator found in life science laboratories. A UV transilluminator or 'light box' is typically used to view gels containing DNA samples. Due to the bright UV sample irradiation requirements, transilluminators are equipped with clear plastic safety covers to attenuate the tissue-damaging UV light and come with explicit instructions for skin and eye protection PPE measures.

Nevertheless, UV transilluminator over-exposure incidents are occurring. In a recent example in 2006, a DOE employee received first degree sunburn symptoms to the face after working on a transilluminator with the lid open for only 3 minutes [6]. In this incident the worker wore UV protective eyewear and latex gloves but no protective face shield. Subsequent measurements indicated UV exposure levels of $200 \mu \mathrm{W} / \mathrm{cm}^{2} 18$ inches from the source without the protective lid in place and $<0.02 \mu \mathrm{W} / \mathrm{cm}^{2}$ at the lid position with the lid in place. This difference in source irradiance due to the lid attenuation was a factor of $10^{4}$. With the lid open the Threshold Limit Value (TLV) was exceeded with only 15-second exposure time.

A similar incident at a DOE lab occurred in 2010 when an intern donned protective gloves, a full face-shield and laboratory coat while working again with a UV transilluminator [6]. After an estimated 30 minutes integrated exposure time during a single day, 'sunburn' conditions developed on the lower neck area left exposed by the face shield. Subsequent measurements indicated the UV intensity was of order $20 \mu \mathrm{W} / \mathrm{cm}^{2}$ for which the maximum exposure time is only 2.5 minutes. Although the injuries were minor, further investigation found a Hazard Awareness Summary document had not been filed so permits, hazard identification, and engineering and administrative controls were not in place.

In a third more graphical UV transilluminator case, several undergraduate students received corneal eye injuries in an introductory science laboratory [6]. In this incident some of the students had performed work without the protective lid in place while others had performed work without protective UV goggles altogether. Fortunately after hospital treatment the resulting eye injuries healed without chronic symptoms. The root cause of the incident was identified as lack of instructions in the lab manuals calling for use of the UV transilluminator shield or skin/eye protection. All three of these cases point to the common symptom of personnel not fully aware of the hazards associated with UV light exposure.

Skin injuries are also a concern with high power industrial lasers. In one documented incident at a DOE lab a technician received second degree burns to the hand while inserting a target in a Class $4 \mathrm{CO}_{2}$ laser beam during an alignment procedure [5]. Knowing the inherent dangers of the systems, the technical team followed lockout/tagout procedures and carefully 
followed explicit steps specified in the work order form. Nevertheless, when the system was re-energized the technician incorrectly believed the system was in 'simmer' mode with no laser beam present and without performing zero-energy verification attempted to place a target in the beam path. Instead the laser was operating at full power resulting in $2^{\text {nd }}$-degree burns to the tips of two fingers. As stated in the incident report 'all personnel are fallible and even the best will make mistakes’.

As these examples illustrate laser radiation and other intense UV sources can present unforeseen skin hazards in research and industrial environments. A common element found in many UV skin and eye injury cases is that the invisible radiation and deceptively low MPE hazards are not fully appreciated. To help mitigate the inherent hazards, excellent educational guidance documents can be found in the literature (an example for UV transilluminator operation can be found in [8]). General guidance for UV protection includes installing proper shielding at the source, donning protective eyewear and skin protection, and reducing exposure time.

\section{Guidance for Protecting Skin from Hazardous Exposures to Class 3B and Class 4 UV Laser Radiation}

Laser personnel need to be aware that high power lasers have significant potential to be a skin hazard as well as an eye hazard. To illustrate the danger skin PPE recommendations are given in Table 2 for the example conditions summarized in Table 1. Eye PPE should be mandatory whenever there may be accessible Class 3B or Class 4 laser beams, including all the conditions presented in Table 2.

Additional guidance for skin protection:

- $\quad$ Engineering controls (enclosures and barriers)

o Enclose UV laser beam paths to extent practical. If the radiation cannot be enclosed, then implement adequate barriers to minimize potential exposure to the direct beam, and to beam losses and other sources of diffuse reflections.

o Beam dumps. Design barriers or enclosures for beam dumps to minimize potential exposure to diffuse UV reflections.

- Administrative procedures

o Attenuate laser beam to minimum power required when there are open beams, in particular when alignment is done. o Use remote steering controls and diagnostics as much as practical for aligning UV beams.

o Plan work to minimize time with potential skin exposure to hazardous UV beams.

o Keep exposed skin as far as practical from open beams.

- $\quad$ PPE for skin

o Wear long-sleeved shirts.

o Use gloves when working with hands near accessible laser beams (direct beam exposure hazard for primary or stray beams).

o Use gloves when the diffuse reflection NHZ is greater than $20 \mathrm{~cm}$ if hands may be within this distance of an open beam path when diffuse reflections may not be well shielded.

o Use face shield when the diffuse reflection NHZ is greater than $1 \mathrm{~m}$ if working within this distance of an open beam path when diffuse reflections may not be well shielded.

- Medical exams

o Periodic skin exams are recommended for laser personnel who may have chronic exposures exceeding MPE values.

- $\quad$ Site-specific training

o On-the-Job Training (OJT) and the Standard Operating Procedures (SOP) document need to describe the potential for skin injury and controls to use. These need to emphasize barriers and enclosures for UV beams and when to use skin PPE.

Table 2: Skin PPE recommendations for conditions considered in Table 1, if working near an open UV laser beam when diffuse reflections are not well shielded.

\begin{tabular}{|c|c|c|c|c|}
\hline $\begin{array}{c}\text { Wavelength } \\
\text { (nm) }\end{array}$ & $\begin{array}{c}\text { Avg. } \\
\text { Power } \\
\text { (W) }\end{array}$ & NHZ & $\begin{array}{c}\text { Use } \\
\text { Gloves? }\end{array}$ & $\begin{array}{c}\text { Use } \\
\text { Face } \\
\text { shield? }\end{array}$ \\
\hline $315-400$ & $>90$ & $>1 \mathrm{~m}$ & Yes & Yes \\
\hline $315-400$ & $>3.5$ & $>20 \mathrm{~cm}$ & Yes & - \\
\hline $190-315$ & $>0.25$ & $>1 \mathrm{~m}$ & Yes & Yes \\
\hline $190-315$ & $>0.01$ & $>20 \mathrm{~cm}$ & Yes & - \\
\hline
\end{tabular}




\section{Summary}

Skin injuries from UV laser operation are not well documented, but are a significant concern. MPEs in the UV depend on the cumulative dose, and potential extended exposures to diffuse radiation need to be considered. These MPEs are the same for coherent and incoherent sources and we've described some recent UV skin injury reports from transilluminator sources. Skin protection can best be achieved with engineering controls and UV laser beam paths should be enclosed as much as practical. Administrative controls during alignment or open beam operation should include using the minimum beam power needed and utilizing remote steering controls and diagnostics. PPE should include long-sleeved shirts, gloves when hands are close to direct beams or if the NHZ from diffuse reflections can be greater than $20 \mathrm{~cm}$, and face shields if the NHZ from diffuse reflections can be greater than $1 \mathrm{~m}$.

\section{Acknowledgements}

Work supported by the U.S. Department of Energy under contract number DE-AC02-76SF00515.

\section{References}

[1] American National Standard for Safe Use of Lasers, ANSI Z136.1-2007, published by Laser Institute of America, http://www.lia.org/. [2] OSHA Technical Manual on Laser Safety, www.osha.gov/dts/osta/otm/otm_iii/otm_iii_6.html. [3] ACGIH 2011 Threshold Limit Values (TLVs) and Biological Exposure Indices (BEIs), published by ACGIH, http://www.acgih.org/TLV/.

[4] EasyHaz laser hazard analysis software, EasyHaz $^{\mathrm{TM}}$ Professional v4.0.

[5] Eye and skin injury reports from laser operation at DOE labs between 2001-2012 are described in the following documents:

- $\quad$ SOR 2005-1, Special Operations Report: Laser Safety, describes 6 eye injury incidents between 2001-2005;

http://www.efcog.org/wg/esh_ls/docs/ORPSLaser/Laser_Safety_Report.pdf.

- Two lessons learned reports, Lessons Learned DOE-SLAC-2009 Laser Injury Accident and Lessons Learned DOE-INL-2011 Hand Injury Report, describe an eye injury in 2009 and a hand injury in 2011. These reports are linked from www.efcog.org/wg/esh_ls/documents.htm.
[6] Recent reports on eye and skin injuries from exposures to incoherent UV transilluminator light sources:

- 2006 skin burn at Argonne National Lab, DOE Lessons Learned report 2006-ANL-004, "First Degree Sunburn from UV Transilluminator."

- 2010 skin burn at Pacific Northwest National Lab, DOE ORPS Report SC-PNSO-PNNLPNNLBOPER-2010-0014, "Researcher

Receives Threshold Limit Value UV Exposure."

- 2011 eye injuries in a Harvard lab course are described in this article, http://www.thecrimson.com/article/2011/12/5 /LPSA-Mishap-Investigation/.

[7] Rockwell Laser Industries Accident Database, http://www.rli.com/resources/accident.aspx.

[8] Guidance document on safety for UV transilluminators from the University of Edinburgh, http://www.docs.csg.ed.ac.uk/Safety/rpu/cop/RP_COP 202.pdf.

\section{Meet the Authors}

Jeff Corbett is the Deputy Laser Safety Officer at the SLAC National Accelerator Laboratory. His background is in Electrical Engineering where he earned a PhD in Nuclear Fusion and Plasma Physics from the University of California at Los Angeles. He has worked 23 years at SLAC on accelerator operations, machine design, beam feedback and diagnostic systems utilizing both electron and photon beams. He presently serves as the System Laser Safety Officer for photocathode research facilities at SSRL and the ASTA laboratory at SLAC.

Michael Woods, CLSO, is the Laser Safety Officer at the SLAC National Accelerator Laboratory. He is an Engineering Physicist, with a B.Sc. in Engineering Physics from Queen's University in Kingston, Ontario, Canada and a Ph.D. in High Energy Physics from the University of Chicago. He has spent 15 years as a researcher in experimental particle physics and accelerator physics, utilizing high power laser systems for photo-injectors, Compton polarimeters and electron beam diagnostics. He became SLAC LSO in 2008. He is a member of the ANSI Z136 SSC-1, TSC-4 and TSC-5 committees. 


\section{Appendix 1: ANSI Z136.1-2007}

The following sections are taken from Reference [1]. ${ }^{2}$ Some sentences are put in italicized boldface for added emphasis.

4.6.2.2 UV Laser Protection. Particular care shall be taken when using UV lasers or laser systems. Thus, in addition to other laser controls which apply to all laser systems, the following requirements shall also apply. Exposure to UV radiation shall be minimized by using beam shields and clothing which attenuate the radiation to levels below the applicable MPE for the specific UV wavelengths. Hazardous byproducts: Special attention shall be given to the possibility of producing undesirable reactions in the presence of UV radiation. For example, formation of skin sensitizing agents, ozone, LGACs, etc. (see Section 7.4). Personal Protective Equipment (PPE) shall be used when working with open beam Class $3 B$ or Class 4 UV lasers. This shall include both eye and skin protection.

4.6.6 Skin Protection (Class 3B or Class 4). In some laser applications, such as use of excimer lasers operating in the ultraviolet, the use of a skin cover shall be employed if chronic (repeated) exposures are anticipated at exposure levels at or near the applicable MPEs for skin. Skin protection can best be achieved through engineering controls. If the potential exists for a damaging skin exposure, particularly for ultraviolet lasers $(0.295-0.400 \mu \mathrm{m})$ and/or laser welding/cutting application, then skin-covers and / or "sun screen" creams are recommended. Most gloves will provide some protection against laser radiation. Tightly woven fabrics and opaque gloves provide the best protection. In some cases a laboratory jacket or coat may fulfill the requirement. For Class 4 lasers, consideration shall be given to flame-retardant materials. For wavelengths greater than $1.4 \mu \mathrm{m}$, "large-area" exposures can cause heat loading causing skin dryness and with excessive exposure, may lead to heat stress (see Section 8.4.2). In these cases, personnel exposures shall be minimized. Chronic exposure may have long term adverse health effects which are not fully understood at this time.

E3.1.1 Preassignment Medical Examinations. Except for examination following suspected injury,

${ }^{2}$ Figure 2 and Sections 4.6.2.2, 4.6.6, E3.1.1, E3.2.6 and G2/G2.1 reproduced with permission from the Z136.1-2007 American National Standard for Safe Use of Lasers. Copyright 2007, Laser Institute of America, Orlando, Florida. The Laser Institute of America disclaims any responsibility or liability resulting from the placement and use in the described manner. these are the only examinations required by this standard. ...Although skin damage from chronic exposure to laser radiation has not been reported, and indeed seems unlikely, this area has not been adequately studied. Limited skin examinations are suggested to serve as a baseline until future epidemiologic studies indicate whether they are needed or not.

E3.2.6 Skin Examination. Not required for preplacement examinations of laser workers; however, it is suggested for employees with history of photosensitivity or working with ultraviolet lasers. Any previous dermatological abnormalities and family history are reviewed. Any current complaints concerned with the skin are noted as well as the history of medication usage, particularly concentrating on those drugs which are potentially photosensitizing. Further examination should be based on the type of laser radiation, above the appropriate MPEs, present in the individual's work environment.

\section{G2. Biological Effects of Laser Radiation on the Skin}

G2.1 General. The large skin surface makes this body tissue readily available to accidental and repeated exposures to laser radiation. The biological significance of irradiation of the skin by lasers operating in the visible and infrared regions is considerably less than exposure of the eye, as skin damage is usually reparable or reversible. Effects may vary from a mild reddening (erythema) to blisters and charring. Depigmentation, ulceration, and scarring of the skin and damage to underlying organs may occur from extremely high-power laser radiation. Outside of the UV region, latent and cumulative effects of laser radiation to the skin are not known at this time. The possibility of such effects occurring, however, should not be ignored in planning for personnel safety in laser installations. Little or no data is available describing the reaction of skin exposed to laser radiation in the 0.2 to $0.4 \mu \mathrm{m}$ spectral region, but chronic exposure to ultraviolet wavelengths in this range can have a carcinogenic action on skin as well as eliciting an erythematous response. On the basis of studies with noncoherent ultraviolet radiation, exposure to wavelengths in the 0.25 to $0.32 \mu \mathrm{m}$ spectral region is most injurious to skin. Exposure to the shorter (0.2 to $0.25 \mu \mathrm{m})$ and longer $(0.32$ to $0.4 \mu \mathrm{m})$ ultraviolet wavelengths is considered less harmful to normal human skin. The shorter wavelengths are absorbed in the outer dead layer of the epidermis (stratum corneum), and exposure to the longer wavelengths has a pigment darkening effect. However, the sensitivity of skin to the longer wavelengths may be increased by known or inadvertent usage of photosensitizers. 


\section{Appendix 2: OSHA Technical Manual on Laser Safety}

The following sections are taken from Reference [2]. Some sentences are put in italicized boldface for added emphasis.

A. OTHER.

1. Other damage mechanisms have also been demonstrated for other specific wavelength ranges and/or exposure times. For example, photochemical reactions are the principal cause of threshold level tissue damage following exposures to either actinic ultraviolet radiation $(0.200 \mu \mathrm{m}$ $0.315 \mu \mathrm{m}$ ) for any exposure time or "blue light" visible radiation $(0.400 \mu \mathrm{m}-0.550 \mu \mathrm{m})$ when exposures are greater than 10 seconds.

2. To the skin, UV-A $(0.315 \mu \mathrm{m}-0.400 \mu \mathrm{m})$ can cause hyperpigmentation and erythema.

3. Exposure in the $U V$-B range is most injurious to skin. In addition to thermal injury caused by ultraviolet energy, there is the possibility of radiation carcinogenesis from $\boldsymbol{U V}-\boldsymbol{B}(\mathbf{0 . 2 8 0} \mathbf{~ m m}$ $0.315 \mathbf{~ m m}$ ) either directly on DNA or from effects on potential carcinogenic intracellular viruses.

4. Exposure in the shorter UV-C $(0.200 \mu \mathrm{m}-0.280$ $\mu \mathrm{m})$ and the longer UV-A ranges seems less harmful to human skin. The shorter wavelengths are absorbed in the outer dead layers of the epidermis (stratum corneum) and the longer wavelengths have an initial pigment-darkening effect followed by erythema if there is exposure to excessive levels. These biological effects are summarized in Table III:6-3.

5. The hazards associated with skin exposure are of less importance than eye hazards; however, with the expanding use of higher-power laser systems, particularly ultraviolet lasers, the unprotected skin of personnel may be exposed to extremely hazardous levels of the beam power if used in an unenclosed system design.

Table 3: Table from Reference [2] summarizing biological effects from optical exposures.

TABLE III:6-3. SUMMARY OF BASIC BIOLOGICAL EFFECTS OF LIGHT

\begin{tabular}{|c|c|c|}
\hline Photobiological spectral domain & Eye effects & Skin effects \\
\hline Ultraviolet C $(0.200-0.280 \mu \mathrm{m})$ & Photokeratitis & $\begin{array}{l}\text { Erythema (sunburn) } \\
\text { Skin cancer }\end{array}$ \\
\hline Ultraviolet B $(0.280-315 \mu \mathrm{m})$ & Photokeratitis & $\begin{array}{l}\text { Accelerated skin aging } \\
\text { Increased } \\
\text { pigmentation }\end{array}$ \\
\hline Ultraviolet A $(0.315-0.400 \mu \mathrm{m})$ & $\begin{array}{l}\text { Photochemical UV } \\
\text { cataract }\end{array}$ & $\begin{array}{l}\text { Pigment darkening } \\
\text { Skin burn }\end{array}$ \\
\hline Visible $(0.400-0.780 \mu \mathrm{m})$ & $\begin{array}{l}\text { Photochemical and } \\
\text { thermal retinal injury }\end{array}$ & $\begin{array}{l}\text { Photosensitive } \\
\text { reactions } \\
\text { Skin burn }\end{array}$ \\
\hline Infrared A $(0.780-1.400 \mu \mathrm{m})$ & Cataract, retinal burns & Skin burn \\
\hline Infrared B $(1.400-3.00 \mu \mathrm{m})$ & $\begin{array}{l}\text { Corneal burn } \\
\text { Aqueous flare } \\
\text { IR cataract }\end{array}$ & Skin burn \\
\hline Infrared C (3.00-1000 $\mu \mathrm{m})$ & Corneal burn only & Skin burn \\
\hline
\end{tabular}




\section{Appendix 3: ACGIH Threshold Limit Values}

The following information on Threshold Limit Values (TLVs) for UV radiation exposure to skin or eye is based on the section on UV radiation in Reference [3].

\section{Broadband UV Sources (180 to $400 \mathrm{~nm}$ ) - Corneal Hazard}

The effective irradiance, $E_{\text {eff }}$, for a broadband UV source is determined from

$$
E_{\text {eff }}=\sum_{180}^{400} E_{\lambda} \cdot S(\lambda) \cdot \Delta \lambda
$$

Where:

- $\quad E_{\text {eff }}$ is the effective irradiance relative to a monochromatic source at $270 \mathrm{~nm}\left[\mathrm{~W} / \mathrm{cm}^{2}\right]$

- $E_{\lambda}$ is the spectral irradiance[W/( $\left.\left.\mathrm{cm}^{2} \cdot \mathrm{nm}\right)\right]$

- $S(\lambda)$ is the relative spectral effectiveness (see Figure 3)

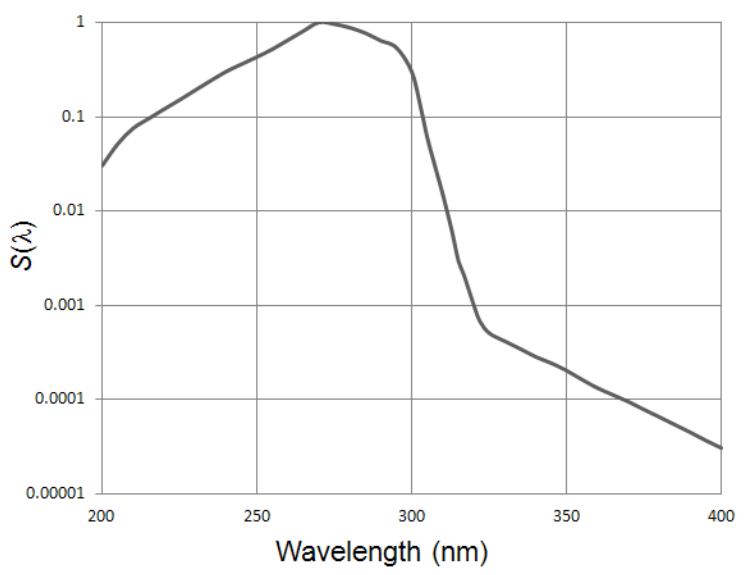

Figure 3: Relative spectral effectiveness, $S(\lambda)$

The daily effective UV exposure dose,

$D_{\text {eff }}^{U V \text {-CornealHazard }}$, is determined by the product of $E_{\text {eff }}$ and the daily exposure time, $t_{\text {exp }}$. To be below the daily TLV, it must be less than $0.003 \mathrm{~J} / \mathrm{cm}^{2}$,

$$
\begin{aligned}
& D_{\text {eff }}^{U V \text {-CornealHazard }}=E_{\text {eff }}\left[\mathrm{W} / \mathrm{cm}^{2}\right] \cdot t_{\text {exp }}[\mathrm{s}] \\
& D_{\text {eff }}^{U V \text {-CornealHazard }} \leq 0.003\left[\mathrm{~J} / \mathrm{cm}^{2}\right]
\end{aligned}
$$

Note: this incoherent source dose limit of $3 \mathrm{~mJ} / \mathrm{cm}^{2}$ is very similar to the MPE of $3 \mathrm{~mJ} / \mathrm{cm}^{2}$ shown in Figure 2 for UV laser exposures between 180-300nm.

\section{Broadband UV-A Sources (315 to $400 \mathrm{~nm}$ ) - Lens and Retinal Hazard}

The UV-A irradiance, $\mathrm{E}_{\mathrm{UV}-\mathrm{A}}\left[\mathrm{W} / \mathrm{cm}^{2}\right]$, is determined from

$$
E_{U V-A}=\sum_{315}^{400} E_{\lambda} \cdot \Delta \lambda
$$

If the daily exposure period is less than 1000s, then to be below the TLV the UV-A exposure dose must be below $1 \mathrm{~J} / \mathrm{cm}^{2}$,

$$
\begin{aligned}
& D^{U V-A}=E_{U V-A}\left[W / \mathrm{cm}^{2}\right] \cdot t_{\exp }[\mathrm{s}] \\
& \text { for } t_{\exp } \prec 1000 \mathrm{~s}, D^{U V-A} \leq 1.0\left[\mathrm{~J} / \mathrm{cm}^{2}\right]
\end{aligned}
$$

If the daily exposure period exceeds 1000 s, then to be below the TLV the UV-A irradiance must be below 1.0 $\mathrm{mW} / \mathrm{cm}^{2}$,

$$
\text { for } t_{\exp } \geq 1000 \mathrm{~s}, E_{U V-A} \leq 1.0\left[\mathrm{~mW} / \mathrm{cm}^{2}\right\rfloor
$$

Note: this incoherent source dose limit of $1 \mathrm{~J} / \mathrm{cm}^{2}$ for daily exposures up to 1000s is the same as the MPE of $1 \mathrm{~J} / \mathrm{cm}^{2}$ shown in Figure 2 for UV laser exposures between 315-400nm.

\section{Additional notes:}

1. Within 40 degrees of the equator, outdoor workers can be exposed to levels above the TLVs in just five minutes at midday during the summer.

2. Ozone is produced in air by UV sources at wavelengths below $250 \mathrm{~nm}$. 